\title{
Certain Spaces of Functions over the Field of Non-Newtonian Complex Numbers
}

\author{
Ahmet Faruk Çakmak ${ }^{1}$ and Feyzi Başar ${ }^{2}$ \\ ${ }^{1}$ Department of Mathematical Engineering, Yıldız Technical University, Davutpaşa Campus, Esenler, 80750 Istanbul, Turkey \\ ${ }^{2}$ Department of Mathematics, Faculty of Arts and Sciences, Fatih University, Hadimköy Campus, Büyükçekmece, \\ 34500 Istanbul, Turkey
}

Correspondence should be addressed to Feyzi Başar; feyzibasar@gmail.com

Received 3 December 2013; Accepted 22 January 2014; Published 15 April 2014

Academic Editor: S.A. Mohiuddine

Copyright (C) 2014 A. F. Çakmak and F. Başar. This is an open access article distributed under the Creative Commons Attribution License, which permits unrestricted use, distribution, and reproduction in any medium, provided the original work is properly cited.

\begin{abstract}
This paper is devoted to investigate some characteristic features of complex numbers and functions in terms of non-Newtonian calculus. Following Grossman and Katz, (Non-Newtonian Calculus, Lee Press, Piegon Cove, Massachusetts, 1972), we construct the field $\mathbb{C}^{*}$ of $*$-complex numbers and the concept of $*$-metric. Also, we give the definitions and the basic important properties of $*$-boundedness and $*$-continuity. Later, we define the space $C_{*}(\Omega)$ of $*$-continuous functions and state that it forms a vector space with respect to the non-Newtonian addition and scalar multiplication and we prove that $C_{*}(\Omega)$ is a Banach space. Finally, Multiplicative calculus (MC), which is one of the most popular non-Newtonian calculus and created by the famous exp function, is applied to complex numbers and functions to investigate some advance inner product properties and give inclusion relationship between $C_{*}(\Omega)$ and the set of $C_{*}^{\prime}(\Omega) *$-differentiable functions.
\end{abstract}

\section{Preliminaries, Background and Notations}

As a popular non-Newtonian calculus, multiplicative calculus was studied by Stanley [1] in a brief overview. Bashirov et al. [2] have recently emphasized on the multiplicative calculus and gave the results with applications corresponding to the well-known properties of derivative and integral in the classical calculus. Recently, in [3], the multiplicative calculus has extended to the complex valued functions and interested in the statements of some fundamental theorems and concepts of multiplicative complex calculus and demonstrated some analogies between the multiplicative complex calculus and classical calculus by theoretical and numerical examples. Bashirov and Riza [4] have studied on the multiplicative differentiation for complex valued functions and established the multiplicative Cauchy-Riemann conditions. Bashirov et al. [5] have investigated various problems from different fields which can be modeled more efficiently using multiplicative calculus, in place of Newtonian calculus. Quite recently, Çakmak and Başar [6] have showed that non-Newtonian real numbers form a field with the binary operations addition and multiplication. Further, the non-Newtonian exponent, surd, and absolute value are defined and some of their properties are given. They also proved that the spaces of all bounded, convergent, null and absolutely p-summable sequences of the non-Newtonian real numbers are the complete metric spaces. Quite recently, Tekin and Başar have [7] proved that the corresponding classical sequence spaces are Banach spaces over the non-Newtonian complex field. Quite recently, Çakir [8] has defined the sets $B(A)$ and $C(A)$ of geometric complex valued bounded and continuous functions and showed that $B(A)$ and $C(A)$ form a vector space with respect to the addition and scalar multiplication in the sense of multiplicative calculus and are complete metric spaces, where $A$ denotes the compact subset of the complex plane $\mathbb{C}$. Quite recently, Uzer [9] has investigated the waves near the edge of a conducting half plane. Firstly, the series is converted into contour integrals in a complex plane and then some contour deformations are made. After that, the resultant integrals are converted back into the series forms, which are seen to be rapidly convergent near the reflection/shadow boundaries of the conducting half plane. In the second part, a multiplicative 
calculus is employed for evaluating the relevant integrals, approximately. By the way, he derives a simple expression, which can be used whenever the series derived in the first part of the paper is not rapidly convergent.

Non-Newtonian calculus is an alternative to the usual calculus of Newton and Leibniz. It provides differentiation and integration tools based on non-Newtonian operations instead of classical operations. Every property in classical calculus has an analogue in non-Newtonian calculus. Generally speaking, non-Newtonian calculus is a methodology that allows one to have a different look at problems which can be investigated via calculus. In some cases, for example, for wage-rate- (in dollars, euro, etc.) related problems, the use of bigeometric calculus which is a kind of nonNewtonian calculus is advocated instead of a traditional Newtonian one.

Throughout this paper, non-Newtonian calculus is denoted by (NC), and classical calculus is denoted by (CC). Also for short we use *-continuity for non-Newtonian continuity. A generator is a one-to-one function whose domain is $\mathbb{R}$ and whose range is a subset of $\mathbb{R}$. Each generator generates exactly one type of arithmetic, and conversely each type of arithmetic is generated by exactly one generator. As a generator, we choose the function exp from $\mathbb{R}$ to the set $\mathbb{R}^{+}$that is to say that

$$
\begin{aligned}
& \alpha: \mathbb{R} \longrightarrow \mathbb{R}^{+} \\
& x \longmapsto \alpha(x)=e^{x}=y, \\
& \alpha^{-1}: \mathbb{R}^{+} \longrightarrow \mathbb{R} \\
& y \\
& y \alpha^{-1}(y)=\ln y=x .
\end{aligned}
$$

In the special cases $\alpha=I$ and $\alpha=\exp , \alpha$ generates the classical and geometric arithmetic, respectively, where $I$ denotes the identity function whose inverse is itself. The set $\mathbb{R}^{*}$ of non-Newtonian real numbers are defined by $\mathbb{R}^{*}:=\{\alpha(x)$ : $x \in \mathbb{R}\}$.

Following Bashirov et al. [2] and Uzer [3], the main purpose of this paper is to construct the space $C_{*}(\Omega)$ of non-Newtonian complex valued continuous functions which forms a Banach space with the norm defined on it. Finally, we give some applications to seek how (NC) can be applied to the classical Functional Analysis problems such as approximation and inner product properties.

We should know that all concepts in classical arithmetic have natural counterparts in $\alpha$-arithmetic. For instance, $\alpha$-zero and $\alpha$-one turn out to be $\alpha(0)$ and $\alpha(1)$. Similarly, the $\alpha$-integers turn out to

$$
\ldots, \alpha(-3), \alpha(-2), \alpha(-1), \alpha(0), \alpha(1), \alpha(2), \alpha(3), \ldots
$$

Consider any generator $\alpha$ with range $A \subseteq \mathbb{R}$. By $\alpha$ arithmetic, we mean the arithmetic whose domain is $A$ and the operations are defined as follows: for $x, y \in A$ and any generator $\alpha$,

$$
\begin{aligned}
& \alpha \text {-addition } x \dot{+} y=\alpha\left[\alpha^{-1}(x)+\alpha^{-1}(y)\right], \\
& \alpha \text {-subtraction } x \dot{x} y=\alpha\left[\alpha^{-1}(x)-\alpha^{-1}(y)\right], \\
& \alpha \text {-multiplication } x \dot{x} y=\alpha\left[\alpha^{-1}(x) \times \alpha^{-1}(y)\right], \\
& \alpha \text {-division } x \dot{y} y=\alpha\left[\alpha^{-1}(x) \div \alpha^{-1}(y)\right], \\
& \alpha \text {-order } x \dot{<} y \Longleftrightarrow \alpha^{-1}(x)<\alpha^{-1}(y) .
\end{aligned}
$$

Particularly, if we choose $I$, the identity function, as an $\alpha$-generator, then $\alpha(x)=x$ and $\alpha^{-1}(x)=x$ for all $x \in A$ and therefore $\alpha$-arithmetic obviously turns out to the classical arithmetic. Consider

$$
\begin{aligned}
\alpha \text {-addition } x \oplus y & =\alpha\left[\alpha^{-1}(x)+\alpha^{-1}(y)\right]=\alpha(x+y) \\
& =x+y \text { classical addition, } \\
\alpha \text {-subtraction } x \ominus y= & \alpha\left[\alpha^{-1}(x)-\alpha^{-1}(y)\right]=\alpha(x-y) \\
= & x-y \text { classical subtraction, } \\
\alpha \text {-multiplication } x \odot y= & \alpha\left[\alpha^{-1}(x) \times \alpha^{-1}(y)\right] \\
= & \alpha(x \times y)=x \times y \\
& \text { classical multiplication, } \\
\alpha \text {-division } x \ominus y= & \alpha\left[\alpha^{-1}(x) \div \alpha^{-1}(y)\right]=\alpha(x \div y) \\
= & x \div y, \quad y \neq 0 \text { classical division. }
\end{aligned}
$$

If we choose exp as an $\alpha$-generator defined by $\alpha(z)=e^{z}$ for $z \in \mathbb{C}$, then $\alpha^{-1}(z)=\ln z$ and $\alpha$-arithmetic turns out to geometric arithmetic. Consider

$$
\begin{aligned}
\alpha \text {-addition } x \oplus y & =\alpha\left[\alpha^{-1}(x)+\alpha^{-1}(y)\right]=e^{(\ln x+\ln y)} \\
& =x \cdot y \text { geometric addition, }
\end{aligned}
$$

$\alpha$-subtraction $x \ominus y=\alpha\left[\alpha^{-1}(x)-\alpha^{-1}(y)\right]=e^{(\ln x-\ln y)}$

$$
=x \div y, \quad y \neq 0
$$

geometric subtraction,

$\alpha$-multiplication $x \odot y=\alpha\left[\alpha^{-1}(x) \times \alpha^{-1}(y)\right]=e^{(\ln x \times \ln y)}$

$$
=x^{\ln y}=y^{\ln x}
$$

geometric multiplication,

$$
\begin{aligned}
\alpha \text {-division } x \oslash y & =\alpha\left[\alpha^{-1}(x) \div \alpha^{-1}(y)\right]=e^{(\ln x \div \ln w)} \\
& =x^{1 / \ln y}, \quad y \neq 1 \text { geometric division. }
\end{aligned}
$$


Arithmetic is any system that satisfies the whole of the ordered field axioms whose domain is a subset of $\mathbb{R}$. There are infinitely many types of arithmetic, all of which are isomorphic, that is, structurally equivalent. Nevertheless, the fact that two systems are isomorphic does not exclude their separate usage. In [2], it is shown that each ordered pair of arithmetic give rise to a calculus by a sensible use of the first arithmetic or function arguments and the second arithmetic for function values.

Let $\alpha$ and $\beta$ be arbitrarily selected generators and ( $\alpha$-arithmetic, $\beta$-arithmetic) is the ordered pair of arithmetic. Table 1 may be useful for the notation used in $\alpha$-arithmetic and $\beta$-arithmetic.

Definitions for $\alpha$-arithmetic are also valid for $\beta$-arithmetic. For example, $\beta$-convergence is defined by means of $\beta$ intervals and their $\beta$-interiors.

In the (NC), $\alpha$-arithmetic is used for arguments and $\beta$-arithmetic is used for ranges; in particular, changes in arguments and ranges are measured by $\alpha$-differences and $\beta$-differences, respectively. The operators of the (NC) are applied only to functions with arguments in $A$ and values in $B$.

The isomorphism from $\alpha$-arithmetic to $\beta$-arithmetic is the unique function $\iota$ (iota) which has the following three properties:

(i) $\iota$ is one to one;

(ii) $\iota$ is on $A$ and onto $B$;

(iii) for any numbers $u$ and $v$ in $A$,

$$
\begin{gathered}
\iota(u \dot{+} v)=\iota(u) \ddot{+} \iota(v), \\
\iota(u \dot{-} v)=\iota(u) \ddot{-} \iota(v), \\
\iota(u \dot{\times} v)=\iota(u) \ddot{\times} \iota(v), \\
\iota(u \dot{/ v})=\iota(u) \ddot{/} \iota(v), \quad v \neq \dot{0}, \\
u \dot{\leq} v \Longleftrightarrow \iota(u) \ddot{\leq} \iota(v) .
\end{gathered}
$$

It turns out that $\iota(x)=\beta\left\{\alpha^{-1}(x)\right\}$ for all $x$ in $A$ and that $\iota(\dot{n})=$ $\ddot{n}$ for every integer $n$.

Since, for example, $u \dot{+} v=\iota^{-1}\{\iota(u) \ddot{+} \iota(v)\}$, it should be clear that any statement in $\alpha$-arithmetic can readily be transformed into a statement in $\beta$-arithmetic.

Throughout this paper, we define the $\dot{p}$-th $*$-exponent $x^{\dot{p}}$ and the $\dot{q}$-th $*$-root $\sqrt[\dot{q}]{x}$ of $x \in \mathbb{R}^{*}$ by

$$
\begin{aligned}
& x^{\dot{2}}=x \dot{\times} x=\alpha\left\{\alpha^{-1}(x) \times \alpha^{-1}(x)\right\}=\alpha\left\{\left[\alpha^{-1}(x)\right]^{2}\right\} \\
& x^{\dot{3}}=x^{\dot{2}} \dot{\times} x=\alpha\left\{\alpha^{-1}\left\{\alpha\left[\alpha^{-1}(x) \times \alpha^{-1}(x)\right]\right\} \times \alpha^{-1}(x)\right\}
\end{aligned}
$$

TABLE 1: Notation in $\alpha$-arithmetic and $\beta$-arithmetic.

\begin{tabular}{lcc}
\hline & $\alpha$-arithmetic & $\beta$-arithmetic \\
\hline Realm & $A$ & $B$ \\
Addition & $\dot{+}$ & $\ddot{+}$ \\
Subtraction & $\dot{-}$ & $\ddot{-}$ \\
Multiplication & $\dot{x}$ & $\ddot{x}$ \\
Division & $\dot{I}$ & $\ddot{~}$ \\
Ordering & $\dot{\leq}$ & $\ddot{\leq}$ \\
\hline
\end{tabular}

$$
\begin{aligned}
= & \alpha\left\{\left[\alpha^{-1}(x)\right]^{3}\right\} \\
& \vdots \\
x^{\dot{p}} & =x^{\dot{p}-1} \dot{\times} x=\alpha\left\{\left[\alpha^{-1}(x)\right]^{p}\right\}
\end{aligned}
$$

and $\sqrt[\dot{q}]{x}=y$ provided there exists an $y \in \mathbb{R}^{*}$ such that $y^{\dot{q}}=x$.

The $\alpha$-absolute value of a number $x$ in $A \subset \mathbb{R}$ is defined as $\alpha\left(\left|\alpha^{-1}(x)\right|\right)$ and is denoted by $|x|$.

For each $\alpha$-nonnegative number $x$, the symbol $\sqrt{x}$ will be used to denote $\alpha\left\{\sqrt{\alpha^{-1}(x)}\right\}$ which is the unique $\alpha$-nonnegative number $y$ whose $\alpha$-square is equal to $x$. For each number $x$ in $A$,

$$
\sqrt{x^{\dot{2}}}=\dot{|x|}=\alpha\left(\left|\alpha^{-1}(x)\right|\right),
$$

where the absolute value $|x|$ of $x \in \mathbb{R}(N)$ is defined by

$$
\dot{|x|}= \begin{cases}x, & x>\alpha(0), \\ \alpha(0), & x=\alpha(0), \\ \alpha(0)-x, & x \dot{<} \alpha(0) .\end{cases}
$$

The $*$-distance between two points $x_{1}$ and $x_{2}$ is defined by $\left|x_{1} \dot{-} x_{2}\right|$ and has the symmetry property, since

$$
\begin{aligned}
\left|x_{1} \dot{-}_{2}\right| & =\alpha\left[\left|\alpha^{-1}\left(x_{1}\right)-\alpha^{-1}\left(x_{2}\right)\right|\right] \\
& =\alpha\left[\left|\alpha^{-1}\left(x_{2}\right)-\alpha^{-1}\left(x_{1}\right)\right|\right] \\
& =\left|x_{2}-x_{1}\right| .
\end{aligned}
$$

Let any $z \in \mathbb{R}^{*}$ be given. Then, $z$ is called a positive non-Newtonian real number if $z>\alpha(0), z$ is called a nonNewtonian negative real number if $z \dot{<} \alpha$ (0), and, finally, $z$ is called an unsigned non-Newtonian real number if $z=\alpha(0)$. By $\mathbb{R}_{+}^{*}$ and $\mathbb{R}_{-}^{*}$, we denote the sets of non-Newtonian positive and negative real numbers, respectively.

In (CC), we have $|x y|=|x||y|$ and $|x+y| \leq|x|+|y|$ for $x, y \in \mathbb{R}$. The following lemmas show that the corresponding results also hold in non-Newtonian calculus.

Lemma 1 ([6, Proposition 2.2]). For any $x, y \in \mathbb{R}^{*},|x \dot{x} y|=$ $\dot{\mid} x \dot{i} \dot{x} \dot{\mid} \mid$. 
Lemma 2 (*-Triangle inequality see [6, Lemma 3.1]). Let $x, y \in \mathbb{R}^{*}$. Then,

$$
|x \dot{+} y| \leq|x| \dot{\mid}|y| .
$$

Let $\left(u_{n}\right)$ be an infinite sequence of the elements in $A$. Then, there is at most one element $u$ in $A$ such that every $\alpha$-interval with $u$ in its $\alpha$-interior contains all but finitely many terms of $\left(u_{n}\right)$. If there is such a number $u$, then $\left(u_{n}\right)$ is said to be $\alpha$-convergent to $u$, which is called the $\alpha$-limit of $\left(u_{n}\right)$. In other words,

$$
u_{n} \longrightarrow u(\alpha \text {-convergent }) \Longleftrightarrow \forall \varepsilon \dot{>} \dot{0},
$$

$\exists n_{0} \in \mathbb{N} \ni\left|u_{n} \dot{-} u\right| \dot{<} \varepsilon \quad \forall n \geq n_{0}$ and some $u \in A$.

The $*$-limit of a function $f$ at an element $a$ in $A$ is, if it exists, the unique number $b$ in $B$ such that, for every infinite sequence $\left(a_{n}\right)$ of arguments of $f$ distinct from $a$, if $\left(a_{n}\right)$ is $\alpha$-convergent to $a$, then $\left\{f\left(a_{n}\right)\right\} \beta$-converges to $b$ and is denoted by $*-\lim _{x \rightarrow a} f(x)=b$. That is,

$$
\begin{gathered}
\lim _{x \rightarrow a}{ }^{*} f(x)=b \Longleftrightarrow \forall \epsilon \ddot{>0}, \\
\exists \delta \dot{>0} \ni \ddot{\mid} f(x) \ddot{-} b \ddot{\mid} \ddot{<} \epsilon \quad \forall x \in A \text { with }|x \dot{-} a| \dot{<} \delta .
\end{gathered}
$$

A function $f$ is $*$-continuous at a point $a$ in $A$ if and only if $a$ is an argument of $f$ and $*-\lim _{x \rightarrow a} f(x)=f(a)$. When $\alpha$ and $\beta$ are the identity function $I$, the concepts of $*$-limit and $*$-continuity are identical with those of classical limit and classical continuity.

The $\beta$-change of a function $f$ over an interval $[a, b]$ is the number $f(b) \ddot{-} f(a)$. A $*$-uniform function is a function in $A$, is $*$-continuous, and has the same $\beta$-change over any two $\alpha$-interval of equal $\alpha$-extent. The $*$-uniform functions are those expressible in the form $\iota\{(m \dot{\times} x) \dot{+} c\}$, where $m$ and $c$ are constants in $A$ and $x$ is unrestricted in $A$. By choosing $m=\dot{i}$ and $c=\dot{0}$, we see that $\iota$ is $*$-uniform. It is characteristic of a $*$-uniform function that, for each $\alpha$-progression of arguments, the corresponding sequence of values is a $\alpha$ progression. The $*$-slope of a $*$-uniform function is its $\beta$ change over any $\alpha$ interval of $\alpha$-extent $\dot{i}$. For example, the *-slope of the function $\iota\{(m \dot{\times} x) \dot{+} c\}$ turns out to be $\iota(m)$. In particular, the $*$-slope of $\iota$ equals $\ddot{1}$, and the $*$-slope of a constant function on $A$ equals 0 .

The $*$-gradient of a function $f$ over $[a, b]$ is the $*$-slope of the $*$-uniform function containing $(a, f(a))$ and $(b, f(b))$ showed as $G_{a}^{* b}$ and turns out to be

$$
G_{a}^{* b}=[f(b) \ddot{-} f(a)] \ddot{l}[\iota(b) \ddot{-} \iota(a)] .
$$

If the following $*$-limit exists, the $*$-derivative of $f\left[D^{*} f\right](a)$ at $a$, and say that $f$ is $*$-differentiable at $a$,

$$
\left[D^{*} f\right](a)=*-\lim _{x \rightarrow a}\{[f(b) \ddot{-} f(a)] \ddot{/}[\iota(b) \ddot{-} \iota(a)]\} .
$$

If it exists, $\left[D^{*} f\right](a)$ is necessarily in $B$.

The $*$-derivative $D^{*} f$ of $f$ is the function that assigns to each number in $A$ the number $\left[D^{*} f\right](t)$, if it exists.
The classical derivatives $[D f](a)$ and $\left[D^{*} f\right](a)$ do not necessarily coexist and are seldom equal; however, if the following exist,

$$
\begin{aligned}
{\left[D\left(\alpha^{-1}\right)\right](a), } & {[D \alpha]\left(\alpha^{-1}(a)\right), } \\
{\left[D\left(\beta^{-1}\right)\right](f(a)), } & {[D \beta]\left\{\beta^{-1}(f(a))\right\}, }
\end{aligned}
$$

then both $[D f](a)$ and $\left[D^{*} f\right](a)$ exist.

We denote the sets of $*$-bounded functions, $*$-continuous functions and $*$-differentiable functions in the $*$-closed interval $[a, b]$ by $\left.\left.B_{*} \dot{[} a, b\right], C_{*} \dot{[} a, b\right]$, and $\left.C_{*}^{\prime} \dot{[} a, b\right]$, respectively.

The $*$-average of a $*$-continuous function $f$ on $[a, b]$ is denoted by $M_{a}^{* b} f$ and defined to be the $\beta$-limit of the $\beta$-convergent sequence whose $n$th term is the $\beta$-average of $f\left(a_{1}\right), \ldots, f\left(a_{n}\right)$, where $a_{1}, \ldots, a_{n}$ is the $n$-fold $\alpha$-partition of $[a, b]$. The $*$-average of a $*$-uniform function on $[a, b]$ is equal to the $\beta$-average of its values at $a$ and $b$ and is equal to its value at the $\alpha$-average of $a$ and $b$.

The $*$-integral $\int_{a}^{* b} f(x) d x$ of a $*$-continuous function $f$ on $[a, b]$, is the following number in $B$ :

$$
[\iota(b) \ddot{-} \iota(a)] \ddot{x} M_{a}^{* b} f .
$$

It is trivial that

$$
\int_{a}^{* a} f(x) d x=\ddot{0} .
$$

Since

$$
\int_{a}^{* b} f(x) d x=M_{a}^{* b}\{[\iota(b) \ddot{-} \iota(a)] \ddot{\times} f\},
$$

the $*$-integral is a weighted $*$-average.

Furthermore, $\int_{a}^{* b} f(x) d x$ equals to the $\beta$-limit of the $\beta$-convergent sequence whose $n$th term is

$$
\left[\iota\left(k_{n}\right) \ddot{\times} f\left(a_{1}\right)\right] \ddot{+} \cdots \ddot{+}\left[\iota\left(k_{n}\right) \ddot{\times} f\left(a_{n-1}\right)\right],
$$

where $a_{1}, \ldots, a_{n}$ is the $n$-fold partition of $\alpha$-partition of $[a, b]$ and $k_{n}$ is the common value of $a_{2} \dot{-} a_{1}, \ldots, a_{n} \dot{-} a_{n-1}$.

If $\alpha$ is classically continuous function and $\beta=I(x)=x$, then the $*$-integral is a Stieltjes integral.

Theorem 3. The *-derivative and *-integral are inversely related in the sense indicated by the following two statements.

(i) If $f$ is *-continuous on $[a, b]$ and $g(x)=\int_{a}^{* x} f(t) d t$ for every $x \in[a, b]$, then $D^{*} g=f$ on $[a, b]$.

(ii) If $D^{*} h$ is $*$-continuous on $[a, b]$, then $\int_{a}^{* b} D^{*} h(x) d x=$ $h(b) \ddot{-h}(a)$.

It is convenient to indicate the uniform relationships between the corresponding notions of the $*$-calculus and classical calculus.

For each number $a \in A$, let $\bar{a}=\alpha^{-1}(a)$. Let $f$ be a function from $A$ into $B$, and set $\bar{f}(t)=\beta\{f(\alpha(t))\}$. 
Then, both $*-\lim _{x \rightarrow a} f(x)$ and $\lim _{t \rightarrow \bar{a}} \bar{f}(t)$ exist and

$$
*-\lim _{x \rightarrow a} f(x)=\beta\left[\lim _{t \rightarrow \bar{a}} \bar{f}(t)\right]
$$

Furthermore, $f$ is $*$-continuous at $a$ if and only if $\bar{f}$ is classically continuous at $\bar{a}$.

If $G_{a}^{* b} f$ is the $*$-gradient of $f$ over $[a, b]$, then $G_{a}^{* b} f=$ $\beta\left\{G_{\bar{a}}^{\bar{b}}\right\} \bar{f}$, where $G_{\bar{a}}^{\bar{b}} \bar{f}$ is the classical gradient of $\bar{f}$ over $\left.\dot{[} a, b\right]$.

If both $\left[D^{*} f\right](a)$ and $D \bar{f}(\bar{a})$ exist, then we have $\left[D^{*} f\right](a)=\beta[D \bar{f}(\bar{a})]$. If $f$ is $*$-continuous on $[a, b]$, then $M_{a}^{* b} f=\beta\left\{M_{\bar{a}}^{\bar{b}}\right\} \bar{f}$ and

$$
\begin{aligned}
\int_{a}^{* b} f & =\beta\left(\int_{\bar{a}}^{* \bar{b}} \bar{f}\right) \\
& =\beta\left\{\int_{\alpha^{-1}(a)}^{\alpha^{-1}(b)} \beta^{-1}[f(\alpha(x))] d x\right\} .
\end{aligned}
$$

The rest of the paper is organized as follows.

In Section 2, it is shown that the set $\mathbb{C}^{*}$ of non-Newtonian complex (*-complex) numbers forms a field with the binary operations addition $(\ddot{+})$ and multiplication $(\ddot{x})$. Further, some basic properties and inequalities which play the basic role in $*$-convergence and $*$-continuity are proved. Section 3 is devoted to the space $C_{*}(\Omega)$ of $*$-continuous functions of a *-complex variable. We prove that $C_{*}(\Omega)$ is a complete metric space with the natural metric and is a Banach space with the natural norm and the space $B^{*}(A ; E)$ of all *-bounded mappings from $A$ into $E$ is a Banach space. As an application part, in Section 4 , we try to create the $*$-inner product space specifically for (MC) and give an inclusion relation between $C_{*}(\Omega)$ and the set of $*$-differentiable functions. In the final section of the paper, we note the significance of the (NC) and record some further suggestions.

\section{2. *-Complex Field and $*$-Inequalities}

In this section, following Tekin and Bașar [7], we give some knowledge on the *-complex field and some concerning inequalities.

Let $\dot{a} \in(A, \dot{+}, \dot{-}, \dot{x}, \dot{l}, \dot{\leq})$ and $\ddot{b} \in(B, \ddot{+}, \ddot{-}, \ddot{x}, \ddot{l}, \ddot{\leq})$ be arbitrarily chosen elements from corresponding arithmetic. Then, the ordered pair $(\dot{a}, \ddot{b})$ is called as a $*$-point. The set of all $*$-points is called the set $*$-complex numbers and is denoted by $\mathbb{C}^{*}$, that is,

$$
\mathbb{C}^{*}:=\left\{z^{*}=(\dot{a}, \ddot{b}): \dot{a} \in A \subseteq \mathbb{R}, \ddot{b} \in B \subseteq \mathbb{R}\right\}
$$

Define the binary operations addition $(\oplus)$ and multiplication $(\odot)$ of $*$-complex numbers $z_{1}^{*}=\left(\dot{a}_{1}, \ddot{b}_{1}\right)$ and $z_{2}^{*}=\left(\dot{a}_{2}, \ddot{b}_{2}\right)$ as follows:

$$
\begin{aligned}
\oplus: \mathbb{C}^{*} \times \mathbb{C}^{*} & \longrightarrow \mathbb{C}^{*} \\
\left(z_{1}^{*}, z_{2}^{*}\right) & \longmapsto z_{1}^{*} \oplus z_{2}^{*}=\left(\dot{a}_{1} \dot{+} \dot{a}_{2}, \ddot{b}_{1} \ddot{+} \ddot{b}_{2}\right), \\
\odot: \mathbb{C}^{*} \times \mathbb{C}^{*} & \longrightarrow \mathbb{C}^{*} \\
\left(z_{1}^{*}, z_{2}^{*}\right) & \longmapsto z_{1}^{*} \odot z_{2}^{*} \\
& =\left(\alpha\left(\overline{\dot{a}}_{1} \overline{\dot{a}}_{2}-\overline{\ddot{b}}_{1} \overline{\vec{b}}_{2}\right), \beta\left(\overline{\dot{a}}_{1} \overline{\vec{b}}_{2}+\overline{\ddot{b}}_{1} \overline{\dot{a}}_{2}\right)\right),
\end{aligned}
$$

where $\dot{a}_{1}, \dot{a}_{2} \in A$ and $\ddot{b}_{1}, \ddot{b}_{2} \in B$ with

$$
\begin{aligned}
& \overline{\dot{a}}_{1}=\alpha^{-1}\left(\dot{a}_{1}\right)=\alpha^{-1}\left(\alpha\left(a_{1}\right)\right)=a_{1} \in \mathbb{R} \\
& \bar{b}_{1}=\beta^{-1}\left(\ddot{b}_{1}\right)=\beta^{-1}\left(\beta\left(b_{1}\right)\right)=b_{1} \in \mathbb{R} .
\end{aligned}
$$

Then, Tekin and Başar [7, Theorem 2.1] proved that $\left(\mathbb{C}^{*}, \oplus, \odot\right)$ is a field.

The $*$-distance $d^{*}$ between any two elements $z_{1}^{*}=\left(\dot{a}_{1}, \ddot{b}_{1}\right)$ and $z_{2}^{*}=\left(\dot{a}_{2}, \ddot{b}_{2}\right)$ of the set $\mathbb{C}^{*}$ is defined by

$$
\begin{aligned}
&\left.d^{*}: \mathbb{C}^{*} \times \mathbb{C}^{*} \longrightarrow \ddot{[} \ddot{0}, \ddot{\infty}\right)=B^{\prime} \subset B \\
&\left(z_{1}^{*}, z_{2}^{*}\right) \longrightarrow d^{*}\left(z_{1}^{*}, z_{2}^{*}\right) \\
&=\sqrt{\left[\iota\left(\dot{a}_{1} \dot{-} \dot{a}_{2}\right)\right]^{\ddot{2}} \ddot{+}\left(\ddot{b}_{1} \ddot{-} \ddot{b_{2}}\right)^{\ddot{2}}} \\
&=\beta\left[\sqrt{\left(a_{1}-a_{2}\right)^{2}+\left(b_{1}-b_{2}\right)^{2}}\right] .
\end{aligned}
$$

Here and after, we know that $\mathbb{C}^{*}$ is a field and the distance between two points in $\mathbb{C}^{*}$ is computed by the relation $d^{*}$. Now, we will see whether this relation $d^{*}$ is metric over $\mathbb{C}^{*}$ or not, define $*$-norm, and try to obtain some required inequalities in the sense of non-Newtonian complex calculus.

$d^{*}\left(z^{*}, \theta^{*}\right)$ is called $*$-norm of $z^{*} \in \mathbb{C}^{*}$ and is denoted by $\ddot{\mid} z^{*} \ddot{\mid}$; that is,

$$
\ddot{\mid} z^{*} \ddot{\mid}=d^{*}\left(z^{*}, \theta^{*}\right)=\sqrt{[\iota(\dot{a} \dot{-} \dot{0})]^{\ddot{2}} \ddot{+}(\ddot{b} \ddot{-} \ddot{0})^{\ddot{2}}}=\beta\left(\sqrt{a^{2}+b^{2}}\right) \text {, }
$$

where $z^{*}=(\dot{a}, \ddot{b})$ and $\theta^{*}=(\dot{0}, \ddot{0})$.

Lemma 4 (*-triangle inequality $\left[7\right.$, Lemma 2.3]). Let $z_{1}^{*}, z_{2}^{*} \in$ $\mathbb{C}^{*}$. Then,

$$
\ddot{\mid} z_{1}^{*} \oplus z_{2}^{*} \ddot{\mid} \ddot{\leq} \ddot{\mid} z_{1}^{*} \ddot{\mid} \ddot{+} \ddot{\mid} z_{2}^{*} \ddot{\mid} \text {. }
$$

Lemma $5\left(\left[7\right.\right.$, Lemma 2.4]). Let $z_{1}^{*}, z_{2}^{*} \in \mathbb{C}^{*}$. Then, $\ddot{\mid} z_{1}^{*} \odot z_{2}^{*} \mid \ddot{\mid}=$ $\ddot{\mid} z_{1}^{*} \ddot{\mid} \ddot{\times} \ddot{\mid} z_{2}^{*} \ddot{\mid}$. 
Lemma 6 (*-Minkowski inequality [7, Lemma 2.5]). Let $p \ddot{\geq} \ddot{1}$ and $z_{k}^{*}, t_{k}^{*} \in \mathbb{C}^{*}$ for all $k \in\{1,2,3, \ldots, n\}$. Then,

$$
\begin{aligned}
\left(\sum_{k=1}^{n} \ddot{\mid} z_{k}^{*} \oplus t_{k}^{*} \ddot{l}^{p}\right)^{1 / p} & \ddot{\leq}\left(\sum_{k=1}^{n} \ddot{\mid} z_{k}^{*} \ddot{p}^{p}\right)^{1 / p} \\
& \ddot{+}\left(\sum_{k=1}^{n} \ddot{\mid} t_{k}^{*} \ddot{\mid}^{p}\right)^{1 / p} .
\end{aligned}
$$

Theorem 7 (see [7, Theorem 2.6]). $\left(\mathbb{C}^{*}, d^{*}\right)$ is a complete metric space, where $d^{*}$ is defined by (27).

In this paper, we mainly focus on the sup metric on the *-complex numbers because $*$-continuity always required to use that metric relation. Therefore, we present the completeness of the set $\mathbb{C}^{*}$ with respect to the sup metric.

Theorem 8. $\mathbb{C}^{*}$ is a Banach space with the norm $\ddot{\|} \cdot \ddot{\|}$ defined by

$$
\ddot{\|} z^{*} \ddot{\|}=\sqrt{[\iota(\dot{a}-\dot{0})]^{\ddot{2}} \ddot{+}(\ddot{b} \ddot{-} \ddot{0})^{\ddot{2}}}
$$

where $z^{*}=(\dot{a}, \ddot{b})$ and $\theta^{*}=(\dot{0}, \ddot{0})$.

\section{Continuous Function Space over the Field $\mathbb{C}^{*}$}

In this section, we construct the space of continuous functions over the field $\mathbb{C}^{*}$ and show that this space is a complete metric space with max metric such that

$$
d^{*}(f, g)=\max _{z \in \mathbb{C}^{*}} \ddot{\mid} f(z) \ddot{-} g(z) \ddot{\mid} .
$$

It would not be too hard to find out that the space of $*$ continuous functions creates a normed space with the norm reduced from the sup metric. Finally, we investigate the completeness property of the spaces of $*$-bounded and $*$ continuous functions.

Let $\Omega \subset \mathbb{C}^{*}$ be compact. Then, by $C_{*}(\Omega)$, we denote the space of $*$-continuous functions defined on the set $\Omega$. One can easily see that the set $C_{*}(\Omega)$ forms a vector space over $\mathbb{C}^{*}$ with respect to the algebraic operations addition $(+)$ and scalar multiplication $(\times)$ defined on $C_{*}(\Omega)$ as follows:

$$
\begin{aligned}
+: & C_{*}(\Omega) \times C_{*}(\Omega) \longrightarrow C_{*}(\Omega) \\
& (f, g) \longmapsto(f+g)(z)=f(z) \ddot{+} g(z), \\
& f=f(z), \quad g=g(z) \in C_{*}(\Omega), \\
\times & \mathbb{C}^{*} \times C_{*}(\Omega) \longrightarrow C_{*}(\Omega) \\
& (\alpha, f(z)) \longmapsto(\alpha \times f)(z)=\alpha \ddot{\times} f(z), \\
& z^{*}=\left(z_{k}^{*}\right) \in \omega^{*}, \quad \alpha \in \mathbb{C}^{*} .
\end{aligned}
$$

In order to show that $C_{*}(\Omega)$ is a metric space with the metric $d^{*}$ defined by (32), we give the following lemma.
Lemma 9. $\left(C_{*}(\Omega), d^{*}\right)$ is a metric space.

Proof. Let $\alpha$ and $\beta$ be the generators on the sets of arguments and values, respectively.

(i) For every $f, g \in C_{*}(\Omega)$ and for every $z \in \Omega$, we have

$$
\begin{aligned}
d^{*} & (f, g) \\
& =\max _{z \in \Omega} \ddot{\mid} f(z) \ddot{-} g(z) \ddot{\mid}=\max _{z \in \Omega} \beta\left\{\left|\beta^{-1}[f(z) \ddot{-g}(z)]\right|\right\} \\
& =\max _{z \in \Omega} \beta\left\{\left|\beta^{-1}\left(\beta\left\{\beta^{-1}[f(z)]-\beta^{-1}[g(z)]\right\}\right)\right|\right\} \\
& =\max _{z \in \Omega} \beta\left\{\left|\beta^{-1}[f(z)]-\beta^{-1}[g(z)]\right|\right\} \\
& =\ddot{0} \Longleftrightarrow f=g ;
\end{aligned}
$$

that is, (M1) holds.

(ii) One can easily see for every $f, g \in C_{*}(\Omega)$ that

$$
\begin{aligned}
d^{*} & (f, g) \\
& =\max _{z \in \Omega} \beta\left\{\left|\beta^{-1}[f(z)]-\beta^{-1}[g(z)]\right|\right\} \\
& =\max _{z \in \Omega} \beta\left\{\left|\beta^{-1}[g(z)]-\beta^{-1}[f(z)]\right|\right\} \\
& =\max _{z \in \Omega} \ddot{g} g(z) \ddot{-} f(z) \ddot{\mid}=d^{*}(g, f),
\end{aligned}
$$

which shows that the symmetry axiom (M2) also holds.

(iii) By a routine verification for every $f, g, h \in C_{*}(\Omega)$, if we apply $\beta^{-1}$ to $\ddot{\mid} f(z) \ddot{-} g(z) \ddot{\mid}$

$$
\begin{gathered}
\begin{aligned}
d^{*}(f, g) & =\max _{z \in \Omega} \ddot{\mid} f(z) \ddot{-} g(z) \ddot{\mid} \\
= & \max _{z \in \Omega} \beta\left\{\left|\beta^{-1}[f(z)]-\beta^{-1}[g(z)]\right|\right\}
\end{aligned} \\
u=\ddot{\mid} f(z) \ddot{-} g(z) \ddot{\mid}=\beta\left\{\left|\beta^{-1}[f(z)]-\beta^{-1}[g(z)]\right|\right\},
\end{gathered}
$$

then we obtain that

$$
\begin{aligned}
& \beta^{-1}(u) \\
&=\left|\beta^{-1}[f(z)]-\beta^{-1}[g(z)]\right| \\
&=\left|\beta^{-1}[f(z)]-\beta^{-1}[h(z)]+\beta^{-1}[h(z)]-\beta^{-1}[g(z)]\right| \\
& \leq\left(\beta \circ \beta^{-1}\right)\left|\beta^{-1}[f(z)]-\beta^{-1}[h(z)]\right| \\
&+\left(\beta \circ \beta^{-1}\right)\left|\beta^{-1}[h(z)]-\beta^{-1}[g(z)]\right|
\end{aligned}
$$




$$
\begin{aligned}
= & \beta^{-1}\left(\beta\left\{\mid \beta^{-1}[f(z)]-\beta^{-1}[h(z) \mid\}\right)\right. \\
& +\beta^{-1}\left(\beta\left\{\mid \beta^{-1}[h(z)]-\beta^{-1}[g(z) \mid\}\right)\right. \\
= & \beta^{-1}(\ddot{\mid} f(z) \ddot{-} h(z) \ddot{\mid}) \\
& +\beta^{-1}(\ddot{\mid} h(z) \ddot{-} g(z) \ddot{\mid}),
\end{aligned}
$$

which yields by applying $\beta$ that

$$
\begin{aligned}
u & \leq \beta\left\{\beta^{-1}(\ddot{\mid} f(z) \ddot{-} h(z) \ddot{\mid})+\beta^{-1}(\ddot{\mid} h(z) \ddot{-} g(z) \ddot{\mid})\right\} \\
& =\ddot{\mid} f(z) \ddot{-} h(z) \ddot{\mid} \ddot{\mid} \ddot{\mid} h(z) \ddot{-} g(z) \ddot{\mid} .
\end{aligned}
$$

Therefore, by taking maximum, one can derive that

$$
\begin{aligned}
d^{*}(f, g) & =\max _{z \in \Omega} \ddot{\mid} f(z) \ddot{-} g(z) \ddot{\mid} \\
& \ddot{\leq} \max _{z \in \Omega} \ddot{\mid} f(z) \ddot{-} h(z) \ddot{\mid} \ddot{+} \max _{z \in \Omega} \ddot{\mid} h(z) \ddot{-g} g(z) \ddot{\mid} \\
& =d^{*}(f, h) \ddot{+} d^{*}(h, g) .
\end{aligned}
$$

This means that the triangle inequality (M3) also holds.

Therefore, since (i)-(iii) are satisfied, $d^{*}$ is a metric on $C_{*}(\Omega)$. This completes the proof.

Definition 10. A $*$-norm is a nonnegative $*$-real valued function on $\Omega \subset \mathbb{C}^{*}$ whose value at an $x \in \Omega$ is denoted by $\|x\|^{*}$, that is, $\|\cdot\|^{*}: \Omega \rightarrow \mathbb{R}$, and satisfies the following conditions:

(N1) $\|x\|^{*}=\ddot{0} \Leftrightarrow x=\ddot{0}$,

(N2) $\|\lambda \ddot{\times} x\|^{*}=\ddot{\mid} \lambda \mid \ddot{\mid} \dot{\times}\|x\|^{*}$ (absolute homogeneity),

(N3) $\|x \ddot{+} y\|^{*} \ddot{\leq}\|x\|^{*} \ddot{+}\|y\|^{*}$ (triangle inequality),

for all $x, y \in \Omega$ and for all scalars $\lambda$. by

The $*$-norm on $C_{*}(\Omega)$ defines a metric $d^{*}$ on $C_{*}(\Omega)$ given

$$
d^{*}(f, g)=\|f \ddot{-g}\|^{*}, \quad f, g \in C_{*}(\Omega),
$$

and is called the induced $*$-metric by the $*$-norm.

The definition of space of continuous functions makes it possible to give a much more intuitive meaning to the classical notion of uniform convergence. Convergence in the space of continuous functions space turns into the uniform convergence. One of the most important results of the concept of the space of continuous functions is the famous Stone-Weierstrass approximation theorem which is a very powerful tool for proof of general results on continuous functions. Using this theorem, we can prove some results fits for functions of special type and later extend them to all continuous functions by a density argument. In this paper, we show, with the rules of non-Newtonian calculus, its advantages to Stone-Weierstrass theorem in the space of $C_{*}(\Omega)$, or not. The answer of this question is affirmative in some cases, but not every time when we want. In [3], Uzer showed by using multiplicative calculus which is a kind of non-Newtonian calculus that it is more flexible than the classical calculus for Bessel functions in a special domain. We can reproduce more examples for the same situation but we mainly focused on the theoretical properties of the space $C_{*}(\Omega)$.

Theorem 11. $C_{*}(\Omega)$ is a normed space with the norm given by

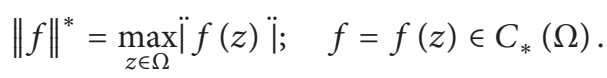

Proof. Let $f, g \in C_{*}(\Omega)$ and $\lambda \in \mathbb{C}$. Then, the following hold.

(i) One can easily show that

$$
\begin{aligned}
\|f\|^{*}=\ddot{0} & \Longleftrightarrow \max \ddot{\mid} f(z) \ddot{\mid}=\ddot{0} \\
& \Longleftrightarrow \ddot{\mid} f(z) \ddot{\mid}=\ddot{0} \quad \forall z \in \Omega \\
& \Longleftrightarrow \beta\left\{\left|\beta^{-1}[f(z)]\right|\right\}=\ddot{0} \quad \forall z \in \Omega \\
& \Longleftrightarrow\left|\beta^{-1}[f(z)]\right|=0 \quad \forall z \in \Omega \\
& \Longleftrightarrow \beta^{-1}[f(z)]=0 \quad \forall z \in \Omega \\
& \Longleftrightarrow f(z)=0 \quad \forall z \in \Omega .
\end{aligned}
$$

That is to say that the axiom (N1) holds.

(ii) From the property of vector space axioms of the space $C_{*}(\Omega)$, it is immediate that

$$
\begin{aligned}
& \|\lambda \ddot{x} f\|^{*}=\max _{z \in \Omega} \ddot{\mid} \lambda \ddot{x} f(z) \ddot{\mid} \\
& =\ddot{\mid} \lambda \ddot{\mid} \dot{\times} \max _{z \in \Omega} \ddot{\mid} f(z) \ddot{\mid} \\
& =\ddot{\mid} \lambda \ddot{\mid} \ddot{x}\|f\|^{*} .
\end{aligned}
$$

Hence, the absolute homogeneity axiom (N2) also holds.

(iii) It is obtained by the similar way used in the proof of Lemma 9 that

$$
\begin{aligned}
\|f \ddot{+} g\|^{*} & =\max _{z \in \Omega} \ddot{\mid} f(z) \ddot{+} g(z) \ddot{\mid} \\
& \ddot{\leq} \max _{z \in \Omega} \ddot{\mid} f(z) \ddot{\mid} \ddot{+} \max _{z \in \Omega} \ddot{\mid} g(z) \ddot{\mid} \\
& =\|f\|^{*} \ddot{+}\|g\|^{*} .
\end{aligned}
$$

This means that the triangle inequality axiom (N3) is satisfied.

Since (i)-(iii) are fulfilled, $\|\cdot\|^{*}$, defined by (41), is a norm for the space $C_{*}(\Omega)$.

Definition 12. Let $A$ be any set and let $E \subset \mathbb{C}^{*}$ be a complex normed space. A mapping $f$ from $A$ into $E$ is bounded if $f(A)$ is bounded in $E$, or equivalently if $\max _{z \in A}\|f(z)\|^{*}$ is finite. The set of all bounded mappings from $A$ into $E$ is denoted by $B^{*}(A ; E)$. 
Corollary 13. The set of all bounded mappings from $A$ into $E$ is denoted by $B^{*}(A ; E)$ is a complex vector space, since

$$
\|f \ddot{+} g\|^{*} \ddot{\leq}\|f\|^{*} \ddot{+}\|g\|^{*} ; \quad f, g \in B^{*}(A ; E) .
$$

Moreover, on this space,

$$
\max _{z \in A}\|f(z)\|^{*}, \quad f \in B^{*}(A ; E),
$$

is a norm, as can be easily verified.

Theorem 14. $B^{*}(A ; E)$ is a Banach space if $E$ is a Banach space.

Proof. Let $\left(f_{n}\right)_{n=0}^{\infty}$ be a Cauchy sequence in $B^{*}(A ; E)$. Then, for any $\varepsilon \ddot{>} \ddot{0}$, there is an $n_{0} \in \mathbb{N}$ such that $\left\|f_{n} \ddot{-} f_{m}\right\|^{*} \ddot{<} \varepsilon$ for all $m, n \geq n_{0}$. From sup norm, it follows for any $z \in A$ that we have $\left\|f_{n}(z) \ddot{-} f_{m}(z)\right\|^{*} \ddot{<} \varepsilon$ for $n, m \geq n_{0}$. Hence, the sequence $\left\{f_{n}(z)\right\}_{n=0}^{\infty}$ converges to an element $f(z) \in E$, since $E$ is complete. Furthermore, we have $\left\|f_{n}(z) \ddot{-} f(z)\right\|^{*} \ddot{<} \varepsilon$ for any $z \in A$ and $n \geq n_{0}$. By the $*$-triangle inequality given by Lemma 4 , we first deduce that $\|f(z)\|^{*} \ddot{<}\left\|f_{n}(z)\right\|^{*} \ddot{+} \varepsilon$ for all $z \in A$; hence $f$ is bounded. Moreover, we have $\left\|f_{n} \ddot{-} f\right\|^{*} \ddot{<} \varepsilon$ for all $n \geq n_{0}$ and this means that the sequence $\left(f_{n}\right)_{n=0}^{\infty}$ converges to $f$ in the space $B^{*}(A ; E)$.

It is known from Mathematical Analysis that when we applied to Non-Newtonian calculus if $\left(f_{n}\right)_{n=0}^{\infty}$ is a sequence of functions from $A$ into a metric space $E$, we say the sequence $\left(f_{n}\right)_{n=0}^{\infty}$ is $*$-convergent to a function $f$ from $A$ into $E$ if, for each $z \in A$, the sequence $\left\{f_{n}(z)\right\}_{n=0}^{\infty} *$-converges in $E$ to $f(z)$; we call that $\left(f_{n}\right)_{n=0}^{\infty} *$-converges uniformly on $A$ to $f$ if the following equality holds:

$$
\lim _{n \rightarrow \infty} \sup _{z \in A} \ddot{\mid} f_{n}(z) \ddot{-} f(z) \ddot{\mid}=\ddot{0} .
$$

It is obvious that $*$-uniform convergence implies $*$-simple convergence; however, the converse is not true. If $E$ is a normed space, then $*$-convergence of a sequence of functions in $B^{*}(A ; E)$, therefore, corresponds to $*$-uniform convergence of the sequence in $A$.

Finally, we give the theorem on the completeness of the space $C_{*}(\Omega)$ of $*$-continuous functions.

Theorem 15. $C_{*}(\Omega)$ is a Banach space with the norm $\|\cdot\|^{*}$ defined by (41).

Proof. Let $\left(f_{n}\right)_{n=0}^{\infty}$ be any Cauchy sequence in $C_{*}(\Omega)$. For each $z \in \Omega$, we have

$$
\begin{aligned}
& \ddot{\mid} f_{n} \ddot{-} f_{m} \ddot{\mid} \ddot{\leq} \mid \ddot{\mid} f_{n}(z) \ddot{-} f_{m}(z) \ddot{\mid}^{*} \\
& \Longleftrightarrow\left|\beta^{-1}\left[f_{n}(z)\right]-\beta^{-1}\left[f_{m}(z)\right]\right| \\
& \leq\left\|\beta^{-1}\left[f_{n}(z)\right]-\beta^{-1}\left[f_{m}(z)\right]\right\|,
\end{aligned}
$$

and so $\left\{f_{n}(z)\right\}$ is a Cauchy sequence of $N$-real numbers, and $\left\{\beta^{-1}\left[f_{n}(z)\right\}\right.$ is a Cauchy sequence of real numbers as well; hence they are $*$-convergent and convergent, respectively. Let $f: \Omega \rightarrow \mathbb{C}^{*}$ be defined by $f(z)=\lim _{n \rightarrow \infty} f_{n}(z)$ or $\beta^{-1}[f(z)]=\lim _{n \rightarrow \infty} \beta^{-1}\left[f_{n}(z)\right]$ for each $z \in \Omega$. Since, given any $\varepsilon>0$,

$$
\left|\beta^{-1}\left[f_{n}(z)\right]-\beta^{-1}\left[f_{m}(z)\right]\right|<\beta^{-1}(\varepsilon)
$$

by letting $n \rightarrow \infty$, we obtain independent of $z$ that

$$
\left|\beta^{-1}[f(z)]-\beta^{-1}\left[f_{m}(z)\right]\right|<\beta^{-1}(\varepsilon),
$$

for sufficiently large $m$. Hence, $\beta^{-1}\left[f_{n}(z)\right] \rightarrow \beta^{-1}[f(z)]$ uniformly in $\Omega$.

Finally, since the limit of a uniformly convergent sequence of continuous functions is continuous, then $f \in$ $C_{*}(\Omega)$ and $f_{n} \rightarrow f$ as $n \rightarrow \infty$. This completes the proof.

\section{Applications}

In this section, we study some properties of multiplicative calculus which is a kind of (NC). This calculus may be created by taking $\alpha=I$, the identity function, and $\beta=\exp$, the exponential function. Multiplicative calculus, in short (MC), has many applications in some branch of mathematics such as financial mathematics and elasticity. In the present paper, we investigate the complex multiplicative functions in a complex domain to make rational approximation for analytic functions. Later, as an application, we mention the inner product property of (MC).

Let $f$ be a single-valued function defined on a set $\Omega$ which is dense itself; that is, every point of $\Omega$ is a limit point of $\Omega$. Then, $f$ is said to be locally analytic on $\Omega$ if, given any $z_{0} \in \Omega$, there is a neighborhood $\aleph\left(z_{0}\right)$ and a power series $\sum_{n=0}^{\infty} a_{n}\left(z-z_{0}\right)^{n}$ such that $f\left(z_{0}\right)=\sum_{n=0}^{\infty} a_{n}\left(z-z_{0}\right)^{n}$ for all $z \in \aleph\left(z_{0}\right) \cap \Omega$. The concept of a locally analytic function on the domain $\Omega$ reduces to a single-valued analytic function.

In (NC), particularly in (MC), when we consider singlevalued analytic functions on a domain, we consider the product, $f\left(z_{0}\right)=\prod_{n=0}^{\infty} a_{n}^{\left(z-z_{0}\right)^{n}}$, where $a_{n}=\left(f^{*(n)}\left(z_{0}\right)\right)^{1 / n !}$ is the $n$th coefficient of the Taylor product of the function $f$ at $z=z_{0}$ such that $f^{*(n)}$ is the $n$th order $*$-derivative of the function $f$.

This is an application to show rational approximation also applicable for $*$-calculus (Multiplicative calculus). One question arises that which way of approximation is better, the classical one or the new one? Uzer made some numerical solutions for the Bessel differential equations in [3] and he considered the function $J_{5}(x+i y)$ with the modulus $\left\|J_{5}(x+i y)\right\|$. It is suggested that if the solution function varies exponentially along a specific contour, then the method in the (MC) sense shows a better performance. Otherwise, that is if the solution function is oscillatory or linearly varying, the method in the (CC) sense will be better. The solution $J_{5}(z)$ in the given example exhibits exponential variations everywhere on the complex plane except near the real axis. The other elements of the family of Bessel functions also exhibit exponential variations. Indeed, there are many other functions exhibiting exponential variations on the complex plane such as the famous sigmoid function $\sigma(z)=\left(1+e^{-z}\right)^{-1}$ 
which plays an important role in decision making in Neutral Networks.

As a second application of (MC), we mention the $*$-inner product property. $\mathrm{A} *$-inner product space $X$ is a vector space with an inner product $\langle z, w\rangle_{*}$ defined on it. A *-norm $\|\cdot\|^{*}$ is defined by $\|z\|^{*}=\sqrt[*]{\langle z, z\rangle_{*}}$ and if $\langle z, w\rangle_{*}=\dot{0}$ holds, then $z$ and $w$ are called $*$-orthogonal vectors. A $*$-Hilbert space $H$ is a complete $*$-inner product space. The spaces to be considered are defined as follows.

Definition 16. Let $X$ be a vector space over the field $\mathbb{C}^{*}$ or $\mathbb{R}^{*}$. A $*$-inner product on $X$ is a mapping from $X \times X$ into the scalar field $K=\mathbb{C}^{*}$ (or $\mathbb{R}^{*}$ ) of $X$; that is, with every pair of vectors $z$ and $w$, there is a scalar $\langle z, w\rangle_{*}$ called the $*$-inner product of $z$ and $w$, such that for all vectors $y, z, w$ and any scalar $\alpha$, the following axioms hold:

$$
\begin{aligned}
& \text { (IP1) }\langle y \dot{+} z, w\rangle_{*}=\langle y, w\rangle_{*} \dot{+}\langle z, w\rangle_{*}, \\
& \text { (IP2) }\langle\alpha \dot{\times} z, w\rangle_{*}=\alpha \dot{\times}\langle z, w\rangle_{*}, \\
& \text { (IP3) }\langle z, w\rangle_{*}=\overline{\langle w, z\rangle_{*}}, \\
& \text { (IP4) }\langle z, z\rangle_{*} \geq \dot{0} \text { and }\langle z, z\rangle_{*}=\dot{0} \Leftrightarrow z=\dot{0} .
\end{aligned}
$$

Then, we say that $X$ is an inner product space provided (IP1)(IP4) hold. Here, $\overline{\langle w, z\rangle_{*}}$ denotes the complex conjugate of $\langle w, z\rangle_{*}$. The conjugate of a $*$-complex number $z=(\dot{x}, \ddot{y})$ is $\bar{z}=(\dot{x},-\ddot{y})$. Note that (IP2) and (IP3) imply that $\langle z, \alpha \dot{\times} w\rangle_{*}=\bar{\alpha} \dot{\times}\langle z, w\rangle_{*}$.

In (MC), the $*$-inner product properties turn into

$$
\begin{aligned}
& (\mathrm{IP}(\mathrm{MC}) 1)\langle y \dot{+} z, w\rangle_{*}=\langle y, w\rangle_{*} \cdot\langle z, w\rangle_{*}, \\
& (\mathrm{IP}(\mathrm{MC}) 2)\langle\alpha \dot{\times} z, w\rangle_{*}=\alpha\langle z, w\rangle_{*}, \\
& (\mathrm{IP}(\mathrm{MC}) 3)\langle z, w\rangle_{*}=\overline{\langle w, z\rangle_{*}}, \\
& (\mathrm{IP}(\mathrm{MC}) 4)\langle z, z\rangle_{*} \geq 1 \text { and }\langle z, z\rangle_{*}=1 \Leftrightarrow z=1 ;
\end{aligned}
$$

then we say that $X$ is multiplicative inner product space.

Corollary 17. It can easily be seen from the equality (24) and from [10] that

$$
\begin{aligned}
& z_{1}^{*} \dot{+} z_{2}^{*}=\left(a_{1} \dot{+} a_{2}, b_{1} \ddot{+} b_{2}\right)=\left(a_{1}+a_{2}, b_{1} \cdot b_{2}\right), \\
z_{1}^{*} \dot{\times} & z_{2}^{*} \\
= & \left(a_{1} \cdot a_{2}-\ln b_{1} \cdot \ln b_{2}, \exp \left\{a_{1} \cdot \ln b_{2}+a_{2} \cdot \ln b_{1}\right\}\right),
\end{aligned}
$$

where $z_{1}=\left(a_{1}, b_{1}\right), z_{2}=\left(a_{2}, b_{2}\right)$ and $\alpha=I, \beta=$ exp. For example, if $z_{1}=(1, e)$ and $z_{2}=\left(1, e^{2}\right)$, then one has

$$
\begin{aligned}
z_{1}^{*} & \dot{\times} z_{2}^{*} \\
& =\left(3 \cdot 1-\ln e \cdot \ln e^{2}, \exp \left\{3 \cdot \ln e^{2}+1 \cdot \ln e\right\}\right)=\left(1, e^{7}\right) .
\end{aligned}
$$

Remark 18. Since the product of $(\dot{0}, \ddot{1})$ with itself equals $(\dot{-} 1,0)$, we may define $i^{*}$ to be $(\dot{0}, \ddot{-} 1)$. Of course, the product of $(\dot{0}, \ddot{-1})$ with itself also equals $(\dot{-} 1, \ddot{0})$. Therefore, in (MC), $i^{*}$ turns out $\left(0, e^{-1}\right)$.
If $z=(x, y)$, then, using again the equalities (24) and $[10$, p. 88$]$, we conclude that

$$
\begin{aligned}
z \dot{\times} \bar{z} & =(\dot{x}, \ddot{y}) \dot{\times}(\dot{x}, \ddot{-} y) \\
& =\left(\beta\left(x^{2}+y^{2}\right), \ddot{0}\right) \\
& =\beta\left(x^{2}+y^{2}\right) \\
& =\ddot{\mid} z \ddot{\mid}^{\ddot{2}} .
\end{aligned}
$$

Thus, in (MC), we have $z \odot \bar{z}=\exp \left(x^{2}+y^{2}\right)$.

In a (real or complex) $*$-inner product space $X$, two vectors $y, z \in X$ are called orthogonal and we write $z \perp w$ provided $\langle z, w\rangle_{*}=\dot{0}$. For a subset $A \subseteq X$, the set $A^{\perp}$ is defined by

$$
A^{\perp}=\left\{u \in X \mid\langle u, z\rangle_{*}=\dot{0} \quad \forall z \in A\right\} .
$$

Corollary 19. A multiplicative inner product space satisfies the parallelogram equality. Let $z=(\dot{x}, \dot{y})$ and $w=(\dot{u}, \dot{v})$ such that $\alpha^{-1}(\dot{x})=x, \alpha^{-1}(\dot{y})=y, \alpha^{-1}(\dot{u})=u, \alpha^{-1}(\dot{v})=v$. Consider

$$
\begin{aligned}
& \ddot{\|} z \ddot{+} w \ddot{\|}^{\ddot{2}}+\ddot{\|} z \ddot{-} w \ddot{\|}^{\ddot{2}}
\end{aligned}
$$

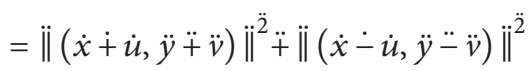

$$
\begin{aligned}
& =(\iota[\dot{x} \dot{+} \dot{u}])^{\ddot{2}},(\ddot{y} \ddot{+} \ddot{v})^{\ddot{2}} \ddot{+}(\iota[\dot{x} \dot{-} \dot{u}])^{\ddot{2}},(\ddot{y}-\ddot{v})^{\ddot{2}} \\
& =\beta\left\{(x+u)^{2}+(y+v)^{2}\right\} \\
& \ddot{+} \beta\left\{(x-u)^{2}+(y-v)^{2}\right\} \\
& =\beta\left\{\beta^{-1}\left[\beta\left\{(x+u)^{2}+(y+v)^{2}\right\}\right]\right. \\
& \left.+\beta^{-1}\left[\beta\left\{(x-u)^{2}+(y-v)^{2}\right\}\right]\right\} \\
& =\beta\left\{(x+u)^{2}+(y+v)^{2}+(x-u)^{2}+(y-v)^{2}\right\} \\
& =\beta\left[2\left(x^{2}+u^{2}+y^{2}+v^{2}\right)\right] \\
& =\beta\left\{2\left[\beta^{-1}\left(\beta\left(x^{2}+y^{2}\right)\right)+\beta^{-1}\left(\beta\left(u^{2}+v^{2}\right)\right)\right]\right\} \\
& =\beta\left\{\beta^{-1}[\beta(2)] \beta^{-1}\left(\ddot{\| z} \ddot{\|}^{2}\right)+\beta^{-1}\left(\ddot{\|} w \ddot{\|}^{2}\right)\right\} \\
& =\beta\left[\beta^{-1}(\ddot{2}) \cdot \beta^{-1}\left(\ddot{\|} z \ddot{\|}^{2} \ddot{+} \ddot{\|} w \ddot{\|}^{2}\right)\right] \\
& =\ddot{2} \ddot{\times}\left[\ddot{\|} z \ddot{\|}^{\ddot{2}} \ddot{+} \ddot{\|} w \ddot{\|}^{2}\right] \text {. }
\end{aligned}
$$

Of course, if we take $\alpha=I$ and $\beta=\exp$, we can easily obtain these results for (MC).

Definition 20. Let $\left(E,\|\cdot\|^{*}\right)$ be a $*$-normed space. If the corresponding metric $d^{*}$ is complete, we say that $\left(E,\|\cdot\|^{*}\right)$ is a Banach space. If $\left(E,\langle\cdot, \cdot\rangle_{*}\right)$ is an $*$-inner product space whose corresponding metric is complete, we say that $\left(E,\langle\cdot, \cdot\rangle_{*}\right)$ is a *-Hilbert space. 
Theorem 21 (Cauchy-Schwartz inequality). For all $z=$ $(\dot{x}, \ddot{y}), w=(\dot{u}, \ddot{v}) \in \mathbb{C}^{*}$, the following inequality holds:

$$
\ddot{\mid}\langle z, w\rangle_{*} \ddot{\mid} \ddot{\leq}\|z\|^{*} \ddot{x}\|w\|^{*},
$$

where $\alpha^{-1}(\dot{x})=x, \alpha^{-1}(\ddot{y})=y, \alpha^{-1}(\dot{u})=u$, and $\alpha^{-1}(\ddot{v})=v$.

Proof. Let $z=(\dot{x}, \ddot{y}), w=(\dot{u}, \ddot{v}) \in \mathbb{C}^{*}$. Then,

$$
\begin{aligned}
\langle z, w\rangle_{*}= & \langle(\dot{x}, \ddot{y}),(\dot{u}, \ddot{v})\rangle_{*} \\
= & \iota(\dot{x} \dot{x} \dot{u}) \ddot{+}(\ddot{y} \ddot{x} \ddot{v}) \\
= & \beta\left\{\alpha^{-1}(\dot{x} \dot{x} \dot{u})\right\} \\
& \ddot{+} \beta\left\{\beta^{-1}(\ddot{y}) \cdot \beta^{-1}(\ddot{v})\right\} \\
= & \beta\left\{\alpha^{-1}\left(\alpha\left\{\alpha^{-1}(\dot{x}) \cdot \alpha^{-1}(\dot{u})\right\}\right)\right\} \\
& \ddot{+} \beta\left\{\beta^{-1}(\ddot{y}) \cdot \beta^{-1}(\ddot{v})\right\} \\
= & \beta\left\{\alpha^{-1}(\dot{x}) \cdot \alpha^{-1}(\dot{u})\right\} \\
& \ddot{+} \beta\left\{\beta^{-1}(\ddot{y}) \cdot \beta^{-1}(\ddot{v})\right\} \\
= & \beta\left\{\alpha^{-1}(\alpha(x)) \cdot \alpha^{-1}(\alpha(u))\right\} \\
& \ddot{+} \beta\left\{\beta^{-1}(\beta(y)) \cdot \beta^{-1}(\beta(v))\right\} \\
= & \beta\{x \cdot u\} \ddot{+} \beta\{y \cdot v\} \\
= & \beta\left\{\beta^{-1}(\beta\{x \cdot u\})+\beta^{-1}(\beta\{y \cdot v\})\right\} \\
= & \beta x \cdot u+y \cdot v\}=\zeta . \\
&
\end{aligned}
$$

If we apply the function of $\beta^{-1}$ to (57), then we have

$$
\begin{aligned}
\beta^{-1}(\zeta) & =x \cdot u+y \cdot v \\
& \leq|x \cdot u|+|y \cdot v| \\
& \leq \sqrt{x^{2} u^{2}+y^{2} v^{2}} \\
& \leq \sqrt{x^{2} u^{2}+y^{2} v^{2}+x^{2} v^{2}+y^{2} u^{2}} \\
& =\sqrt{\left(x^{2}+y^{2}\right)\left(u^{2}+v^{2}\right)} \\
& =\sqrt{x^{2}+y^{2}} \sqrt{u^{2}+v^{2}}
\end{aligned}
$$

which gives by applying $\beta$ that

$$
\begin{aligned}
\langle z, w\rangle_{*} & \ddot{\leq} \beta\left(\sqrt{x^{2}+y^{2}} \sqrt{u^{2}+v^{2}}\right) \\
& =\beta\left\{\beta^{-1}\left[\beta\left(\sqrt{x^{2}+y^{2}}\right)\right] \cdot \beta^{-1}\left[\beta\left(\sqrt{u^{2}+v^{2}}\right)\right]\right\} \\
& =\beta\left(\sqrt{x^{2}+y^{2}}\right) \ddot{x} \beta\left(\sqrt{u^{2}+v^{2}}\right) \\
& =\|(\dot{x}, \ddot{y})\|^{*} \ddot{x}\|(\dot{u}, \ddot{v})\|^{*} \\
& =\|z\|^{*} \ddot{x}\|w\|^{*},
\end{aligned}
$$

which completes the proof.

Theorem 21 gives the following.

Corollary 22. The space $C_{*}(\Omega)$ is an *-inner product space but is not a Hilbert space with the integral metric defined by

$$
\langle f, g\rangle_{*}=\int_{z \in \Omega}^{*} f(z) \overline{g(z)} d z,
$$

where $f, g \in C_{*}(\Omega)$.

The proof is easily obtained by the appropriate verifications. Indeed, if we take $\alpha=I=\beta$, we obtain the classical calculus (CC) and, in (CC), the results are the same for Corollary 22. It is an expected situation, because (CC) is a kind of (NC) and we cannot generalize the assertion of Corollary 22 differently.

Let us consider the space $C[-1,1]$ with the inner product

$$
\langle f, g\rangle=\int_{0}^{1} f(z) \overline{g(z)} d z,
$$

which gives the associated norm $\|f\|=\sqrt{\int_{0}^{1}|f(z)|^{2}} d z$. The inner product space is not complete; the space of Riemannian integrable functions on the interval $[-1 ; 1]$ that are squareintegrable, that is,

$$
\|f\|^{2}=\int_{-1}^{1}|f(z)|^{2} d z<\infty,
$$

is not complete.

As a final application, we give an inclusion relation between the spaces $C_{*}(\Omega)$ and $C_{*}^{\prime}(\Omega)$, the space of first-order $*$-differentiable functions in $\Omega$.

Theorem 23. $C_{*}^{\prime}(\Omega) \subset C_{*}(\Omega)$ and the inclusion is strict.

Proof. In [10], if $f$ is a $*$-continuous function in a given point $a$, then from the definition we have $*-\lim _{x \rightarrow a} f(x)=$ $b=f(a)$ if and only if $\left\{f\left(a_{n}\right)\right\}_{n=0}^{\infty}$ is $\beta$-convergent to $f(a)$ whenever any sequence $\left(a_{n}\right)_{n=0}^{\infty}$ of arguments is $\alpha$-convergent to $a$.

Now, suppose that $f$ is a $*$-differentiable function in a given point $a \in \Omega$. Then, the following limit

$$
*-\lim _{x \rightarrow a}\{[f(x) \ddot{-} f(a)] \ddot{/}[\iota(x) \ddot{-} \iota(a)]\}
$$


exists and is equal to the unique number $\left[D^{*} f\right](a)$. Therefore for every infinite sequence $\left(a_{n}\right)_{n=0}^{\infty}$ of arguments of distinct from $a$, is $\alpha$-convergent to $a$ which implies that $\left[f\left(a_{n}\right) \ddot{-} f(a)\right] \ddot{/}\left[\iota\left(a_{n}\right) \ddot{-} \iota(a)\right]$ is $\beta$-convergent to $\left[D^{*} f\right](a)$ as $n \rightarrow \infty$. It can be written as

$$
\begin{aligned}
{\left[D^{*} f\right](a) } & =*-\lim _{n \rightarrow \infty}\left\{\left[f\left(a_{n}\right) \ddot{-} f(a)\right] \ddot{l}\left[\iota\left(a_{n}\right) \ddot{-} \iota(a)\right]\right\} \\
& =*-\lim _{n \rightarrow \infty}=\beta\left\{\frac{\beta^{-1}\left[f\left(a_{n}\right)\right]-\beta^{-1}[f(a)]}{\beta^{-1}\left[\iota\left(a_{n}\right)\right]-\beta^{-1}[\iota(a)]}\right\} .
\end{aligned}
$$

If we apply $\beta^{-1}$ to $(64)$ and consider the iota function $\iota=\beta$ 。 $\alpha^{-1}$, we obtain that

$$
\begin{aligned}
\beta^{-1} & \left(\left[D^{*} f\right](a)\right) \\
& =\lim _{n \rightarrow \infty} \frac{\beta^{-1}\left[f\left(a_{n}\right)\right]-\beta^{-1}[f(a)]}{\beta^{-1}\left[\iota\left(a_{n}\right)\right]-\beta^{-1}[\iota(a)]} \\
& \Longrightarrow\left(\beta^{-1}\left[\iota\left(a_{n}\right)\right]-\beta^{-1}[\iota(a)]\right) \beta^{-1}\left\{\left[D^{*} f\right](a)\right\} \\
& =\lim _{n \rightarrow \infty}\left\{\beta^{-1}\left[f\left(a_{n}\right)\right]-\beta^{-1}[f(a)]\right\} \\
& \Longrightarrow \lim _{n \rightarrow \infty}\left[\alpha^{-1}\left(a_{n}\right)-\alpha^{-1}(a)\right] \beta^{-1}\left[D^{*} f(a)\right] \\
& =\lim _{n \rightarrow \infty}\left\{\beta^{-1}\left[f\left(a_{n}\right)\right]-\beta^{-1}[f(a)]\right\} .
\end{aligned}
$$

Since $\left(a_{n}\right)$ is $\alpha$-convergent to $a$, the difference of $\alpha^{-1}\left(a_{n}\right)-$ $\alpha^{-1}(a)$ converges to 0 . Therefore, we conclude that

$$
0=\lim _{n \rightarrow \infty}\left\{\beta^{-1}\left[f\left(a_{n}\right)\right]-\beta^{-1}[f(a)]\right\} .
$$

Now, we have by applying $\beta$ to (66) that

$$
\begin{aligned}
\beta(0)=\ddot{0} & =*-\lim _{n \rightarrow \infty} \beta\left\{\beta^{-1}\left[f\left(a_{n}\right)\right]-\beta^{-1}[f(a)]\right\} \\
& =*-\lim _{n \rightarrow \infty}\left[f\left(a_{n}\right) \ddot{-} f(a)\right],
\end{aligned}
$$

which means that $*-\lim _{n \rightarrow \infty} f\left(a_{n}\right)=f(a)$ and this step concludes the proof.

Following Wen [11], we give a counterexample such that there is a nowhere differentiable continuous function constructed by infinite products. Suppose $0<a_{n}<1$ and $p_{n}$ is an even integer for each $n$, and $\sum_{n=1}^{\infty} a_{n}$ is convergent and set $b_{n}=\prod_{k=1}^{n} p_{k}$. If $2^{n} /\left(a_{n} p_{n}\right) \rightarrow 0$ as $n \rightarrow \infty$, then

$$
f(x)=\prod_{n=1}^{\infty}\left(1+a_{n} \sin b_{n} \pi x\right)
$$

is a continuous nowhere differentiable function.

Now, let us consider that the Non-Newtonian $*$-calculus is multiplicative calculus, which means that the generator functions $\alpha$ and $\beta$ are equal to $I(x)=x$ and $\exp (x)=e^{x}$, respectively. Then, the function $f$ defined by Wen [11] as in (68) is

$$
f(x)=\sum_{n=1}^{\infty *}\left(1+a_{n} \sin b_{n} \pi x\right) .
$$

The *-continuity of $f$ given by (69) is obtained from uniform convergence of the function $e^{x}$. Besides, as we already know from [10], and so forth, multiplicative differentiation has a relationship between the classical differentiation such as

$$
f^{*}(x)=\exp [\ln f(x)]^{\prime}=e^{f^{\prime}(x) / f(x)} .
$$

Therefore, this formula does not allow $*$-differentiability to the function (69), too.

\section{Conclusion}

One of the purposes of this work is to extend the classical calculus to the non-Newtonian real calculus for dealing with complex valued functions. Some of the analogies between (CC) and the (NC) are demonstrated by theoretical examples. We derive classical continuous function space in the sense of non-Newtonian calculus and try to understand their structure of being non-Newtonian vector space. Generally, we work on the vector spaces which concern physics and computing. There are lots of techniques that have been developed in the sense of (CC). If (NC) is employed together with (CC) in the formulations, then many of the complicated phenomena in physics or engineering may be analyzed more easily. Even some biological and finance problems can be solved by exponential calculus, which is just a sort of nonNewtonian calculus.

Quite recently, Talo and Başar have studied the certain sets of sequences of fuzzy numbers and introduced the classical sets $\ell_{\infty}(F), c(F), c_{0}(F)$, and $\ell_{p}(F)$ consisting of bounded, convergent, null, and absolutely $p$-summable sequences of fuzzy numbers in [12]. Next, they have defined the $\alpha$-, $\beta$ , and $\gamma$-duals of a set of sequences of fuzzy numbers and gave the duals of the classical sets of sequences of fuzzy numbers together with the characterization of the classes of infinite matrices of fuzzy numbers transforming one of the classical set into another one. Following Bashirov et al. [2] and Uzer [3], we have given the corresponding results for non-Newtonian calculus to the results obtained for fuzzy valued sequences in Talo and Başar [12], as a beginning. As a natural continuation of this paper, we should record that it is meaningful to define the $\alpha-, \beta$-, and $\gamma$-duals of a space of sequences of non-Newtonian real elements and to determine the duals of classical spaces $\ell_{\infty}^{*}, c^{*}, c_{0}^{*}$, and $\ell_{\ddot{p}}^{*}$ together with the characterization of matrix transformations between the classical sequence spaces over the non-Newtonian complex field $\mathbb{C}^{*}$. Further, one can obtain the similar results by using another type of calculus instead of non-Newtonian calculus.

Non-Newtonian calculus is a new area in mathematics and has very pristine subjects to discuss. We just begin with the space of continuous and bounded functions which would step us to investigate more complicated theoretical structures and properties of (NC). We are trying to develop something valuable about non-Newtonian Functional Analysis, but only the mathematical authorities can decide that. 


\section{Conflict of Interests}

The authors declare that there is no conflict of interests regarding the publication of this paper.

\section{Acknowledgments}

The authors have benefited a lot from the referee's report. So, they would like to express their gratitude for his/her constructive suggestions which improved the presentation and readability.

\section{References}

[1] D. Stanley, “A multiplicative calculus," Primus, vol. 9, no. 4, pp. 310-326, 1999.

[2] A. E. Bashirov, E. M. Kurpınar, and A. Özyapıc1, "Multiplicative calculus and its applications," Journal of Mathematical Analysis and Applications, vol. 337, no. 1, pp. 36-48, 2008.

[3] A. Uzer, "Multiplicative type complex calculus as an alternative to the classical calculus," Computers \& Mathematics with Applications, vol. 60, no. 10, pp. 2725-2737, 2010.

[4] A. Bashirov and M. Riza, "On complex multiplicative differentiation," TWMS Journal of Applied and Engineering Mathematics, vol. 1, no. 1, pp. 75-85, 2011.

[5] A. E. Bashirov, E. Mısırlı, Y. Tandoğdu, and A. Özyapıcı, "On modeling with multiplicative differential equations," Applied Mathematics, vol. 26, no. 4, pp. 425-438, 2011.

[6] A. F. Çakmak and F. Başar, "On the classical sequence spaces and non-Newtonian calculus," Journal of Inequalities and Applications, vol. 2012, Article ID 932734, 12 pages, 2012.

[7] S. Tekin and F. Başar, "Certain sequence spaces over the nonNewtonian complex field," Abstract and Applied Analysis, vol. 2013, Article ID 739319, 11 pages, 2013.

[8] Z. Çakır, "Spaces of continuous and bounded functions over the field of geometric complex numbers," Journal of Inequalities and Applications, vol. 2013, article 363, 2013.

[9] A. Uzer, "Exact solution of conducting half plane problems as a rapidly convergent series and an application of the multiplicative calculus," Turkish Journal of Electrical Engineering \& Computer Sciences. In press.

[10] M. Grossman and R. Katz, Non-Newtonian Calculus, Lee Press, Pigeon Cove, Mass, USA, 1972.

[11] L. Wen, "A nowhere differentiable continuous function constructed by infinite products," The American Mathematical Monthly, vol. 109, no. 4, pp. 378-380, 2002.

[12] Ö. Talo and F. Başar, "Determination of the duals of classical sets of sequences of fuzzy numbers and related matrix transformations," Computers \& Mathematics with Applications, vol. 58, no. 4, pp. 717-733, 2009. 


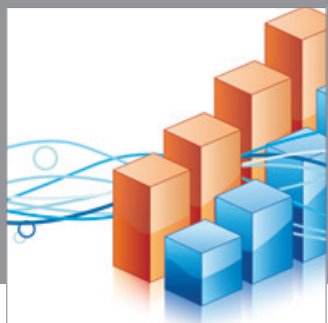

Advances in

Operations Research

mansans

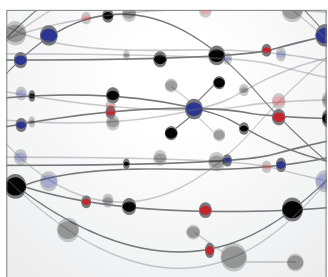

The Scientific World Journal
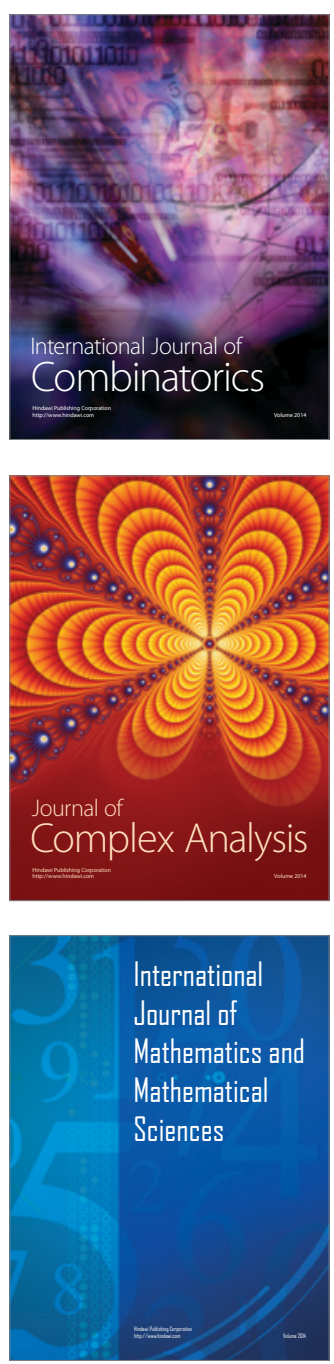
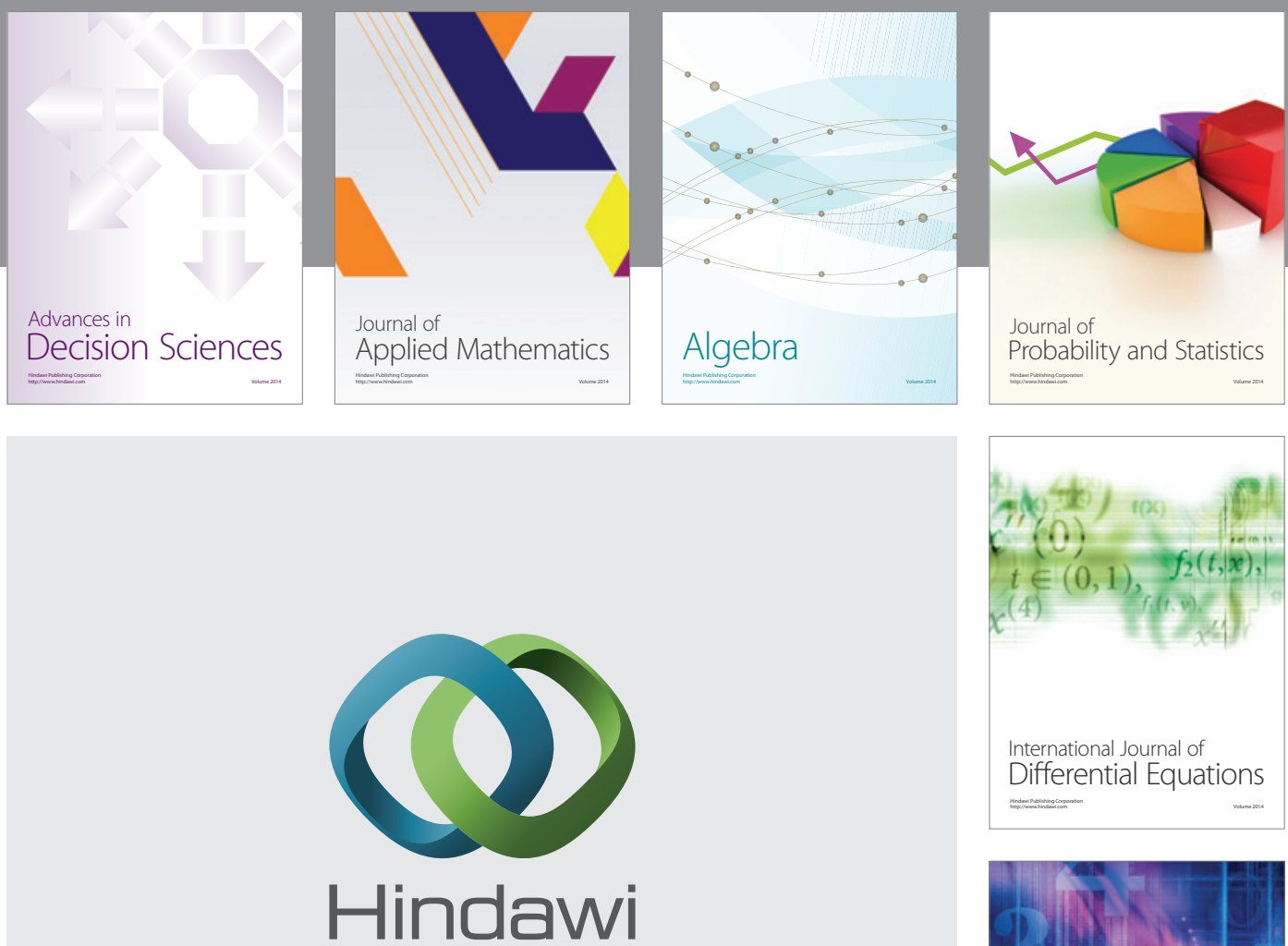

Submit your manuscripts at http://www.hindawi.com
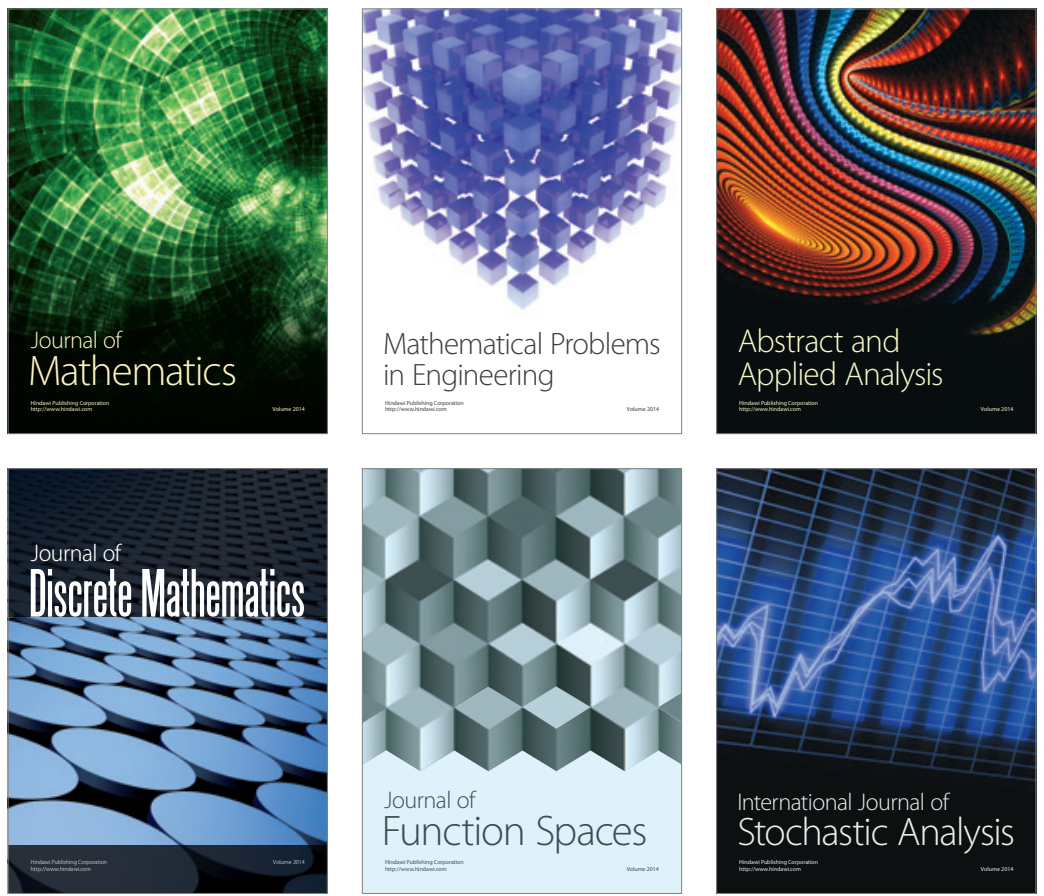

Journal of

Function Spaces

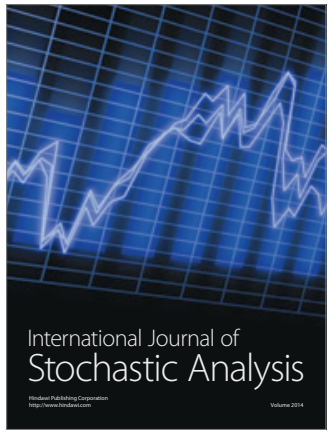

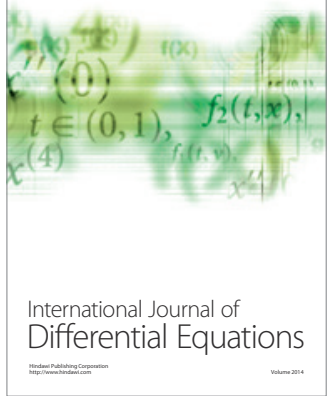
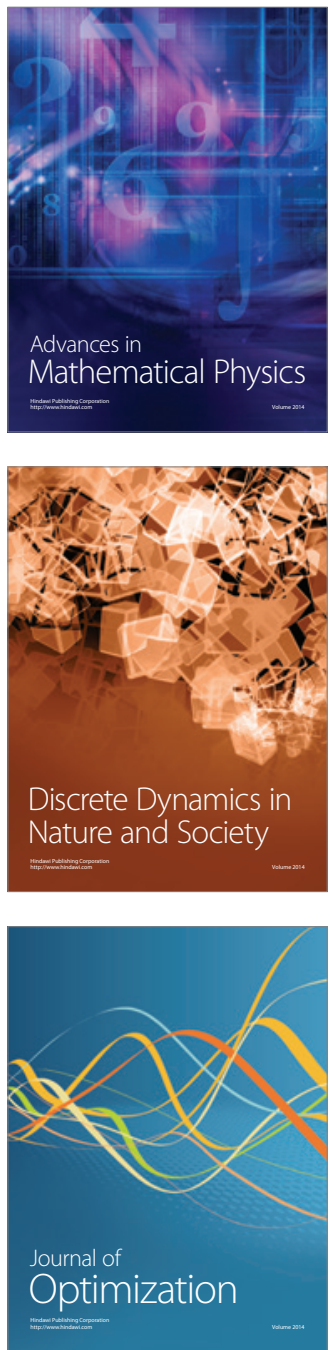